\title{
EphB4 Tyrosine Kinase Stimulation Inhibits Growth of MDA-MB-231 Breast Cancer Cells in a Dose and Time Dependent Manner
}

\author{
Farnaz Barneh, ${ }^{1}$ Mona Moshayedi, ${ }^{1}$ Hamid Mirmohammadsadeghi, ${ }^{2}$ \\ Shaghayegh Haghjooy-Javanmard, ${ }^{1}$ Ali Mohammad Sabzghabaee, ${ }^{3}$ and Shirinsadat Badri ${ }^{4}$ \\ ${ }^{1}$ Applied Physiology Research Center, Isfahan University of Medical Sciences, Isfahan, Iran \\ ${ }^{2}$ Department of Pharmaceutical Biotechnology, School of Pharmacy and Pharmaceutical Sciences, \\ Isfahan University of Medical Sciences, Isfahan, Iran \\ ${ }^{3}$ Isfahan Clinical Toxicology Research Center, Isfahan University of Medical Sciences, Isfahan, Iran \\ ${ }^{4}$ Department of Clinical Pharmacy and Pharmacy Practice, School of Pharmacy and Pharmaceutical Sciences, \\ Isfahan University of Medical Sciences, Isfahan, Iran \\ Correspondence should be addressed to Ali Mohammad Sabzghabaee; sabzghaba@pharm.mui.ac.ir
}

Received 1 August 2013; Revised 3 October 2013; Accepted 20 October 2013

Academic Editor: Yi-Chia Huang

Copyright ( 2013 Farnaz Barneh et al. This is an open access article distributed under the Creative Commons Attribution License, which permits unrestricted use, distribution, and reproduction in any medium, provided the original work is properly cited.

Background. EphB4 receptor tyrosine kinase is of diagnostic and therapeutic value due to its overexpression in breast tumors. Dual functions of tumor promotion and suppression have been reported for this receptor based on presence or absence of its ligand. To elucidate such discrepancy, we aimed to determine the effect of time- and dose-dependent stimulation of EphB4 on viability and invasion of breast cancer cells via recombinant ephrinB2-Fc. Methods. Cells were seeded into multiwell plates and were stimulated by various concentrations of preclustered ephrinB2-Fc. Cell viability was measured on days 3 and 6 following treatment using alamar-blue when cells were in different states of confluence. Results. Stimulation of cells with ephrinB2 did not pose any significant effect on cell viability before reaching confluence, while inhibition of cell growth was detected after 6 days when cells were in postconfluent state following a dose-dependent manner. EphrinB2 treatment did not affect tubular formation and invasion on matrigel. Conclusion. This study showed that EphB4 can differentially inhibit cells at post confluent state and that presence of ligand manifests growth-inhibitory properties of EphB4 receptor. It is concluded that growth inhibition has occurred possibly due to long treatment with ligand, a process which leads to receptor downregulation.

\section{Introduction}

Cancer cells express many factors that enable them to overcome regulatory barriers towards uninterrupted growth and to survive in abnormal circumstances in which normal cells will not [1]. Among such factors, tyrosine kinases play pivotal roles. Eph (Erythropoietin Producing Hepatoma) receptors comprise the largest family of tyrosine kinases and are divided into two classes of EphAs and EphBs based on their sequence homology. Receptor activation occurs via their membrane bound ligands ephrinAs and ephrinBs, respectively [2].
One distinguishing feature of this family is their ability to propagate bidirectional signals in cells expressing receptor and ligand; in other words, both Eph receptors and ephrin ligands are capable of inducing downstream signals in the form of forward and reverse signals [3]. Owing to such spatial orientation, Eph receptors and ephrin ligands construct a communication system between cells expressing the receptor and ligand, which allows them to be involved in many physiological processes such as proliferation, morphology and movement, normal organ out-growth during neuronal path finding, and angiogenesis [4-7]. 
Recently, their involvement in abnormalities such as cancer has been documented as well [8]. EphB4 and EphA2 are the most studied members of this family in cancer. Unlike other tyrosine kinases which are mostly oncogenic, dual functions of tumor promotion and suppression have been reported for this family [8].

EphB4 expressed on cancer cells interacts with ephrinB2 present on endothelial cells and induces angiogenesis [9]. Consistently, soluble EphB4 receptors which are incapable of stimulating endothelial ephrinB2 ligands have been shown to possess therapeutic potential in decreasing tumor growth through inhibition of angiogenesis [10, 11]. Moreover, EphB4 overexpression has been shown to be correlated with increased tumor grade and stage [12] and overexpressing EphB4 receptor in nonmalignant breast and prostate cells leads to their transformation and manifestation of malignant behavior [13]. Accordingly, downregulation of EphB4 decreases breast cancer cells' viability indicating that EphB4 expression serves as a survival factor [14].

Besides EphB4 overexpression in cancer cells, receptor phosphorylation in tumor cells is very low, and it has been shown that ephrinB2, the preferred ligand for EphB4, is downregulated as the receptor is overexpressed [15]. This has led to a hypothesis that ephrinB2 mediated EphB4 activation and a balanced expression of receptor and ligand lead to tumor suppression [16]. This hypothesis was further supported by Noren et al., in which they reported activation of a tumor suppressor signaling pathway, Abl-Crk, downstream of ephrinB2/EphB4 signaling in breast cancer cells [17].

Besides novel studies to date dedicated to characterize functions of Eph receptors in cancer, the complexities associated with their role necessitates more elaborate understanding of their contribution to tumor biology in order to properly target and fully exploit their tumor suppressive behavior in cancer treatment. We believe that fatal effects of ephrinB2 treatment on breast cancer cells may also be time and dosedependent. Herein, we examine the effect of short- and longterm treatment of various ephrinB2-Fc concentrations on growth of MDA-MB-231 breast cancer cells in different states of confluence, and we test the effects of such treatments on aggressive behavior of cancer cells in a three-dimensional culture with matrigel.

\section{Material and Methods}

2.1. Reagents. EphrinB2-Fc was purchased from R\&D Systems. Fc fragment and goat antihuman IgG for clustering ephrinB2-Fc were obtained from Jackson ImmunoResearch. Alamar-blue was obtained from AbdSerotec, and matrigel was purchased from Invitrogen.

2.2. Cell Culture. MDA-MB-231 breast cancer cell line was obtained from Pasteur Institute of Iran. Cells were maintained in DMEM supplemented with 10\% FBS plus 1\% penicillin/streptomycin and were kept in humidified 5\% $\mathrm{CO}_{2}$ incubator with medium renewal every 2-3 days. Cells were subcultured before reaching confluence using $0.25 \%$ trypsinEDTA.
2.3. Standardization of Alamar-Blue Viability Assay. Alamarblue is a commercially available solution of resazurin salt developed to measure viability and proliferation of various cultured cells. Unlike tetrazolium salts (such as MTT), alamar-blue dye is water soluble which obviates further crystal solubilization by addition of DMSO. Moreover, alamarblue is not toxic to cells and allows repeated measurements of cell viability over a long period of time making kinetic studies possible. Alamar-blue is converted to a reduced form by mitochondrial enzymes, and the process is accompanied by a change in color from indigo blue to pink, which can be measured by colorimetric or fluorometric reading.

Due to the difference in metabolic capacity of cells in reducing alamar-blue dye, optimum length of incubation and number of cells for plating are two critical variables to be determined before performing viability/cell proliferation assay for each cell line used [18].

Cells growing in the log phase of growth were harvested by trypsin-EDTA, and an initial concentration of $1 \times 10^{6}$ and further dilutions were made and seeded into wells of a 96well plate $(200 \mu \mathrm{L}$ for each well). The medium without cells was used as negative control, and the plate was incubated for $4 \mathrm{~h}$ to allow cell attachment. Twenty microliters of alamarblue was added to each well. The absorbance was measured immediately and every 2 hours at 570 and $600 \mathrm{~nm}$ for the first 6 hours using PowerwaveXS plate reader. The plate was also incubated for the following 24 hours to measure the maximum dye reduction. $\mathrm{pH}$ was strictly controlled at 7-7.4 by keeping the plate in a $\mathrm{CO}_{2}$ incubator during the test.

Percentage reduction of alamar-blue is typically calculated using equation (1)

Percent reduction of alamar-blue

$$
=\frac{(117216 \times A 1)-(80586 \times A 2) \times 100}{(155677 \times N 2)-(14652 \times N 1)}
$$

$A 1$ and $A 2$ are recorded absorbance of test wells at 570 and $600 \mathrm{~nm}$, respectively, and $N 1$ and $N 2$ are observed absorbance of negative control.

Alternatively, an online calculator suggested by providing company was used for measuring percentage reduction (http://www.abdserotec.com/).

2.4. Alamar-Blue Assay for Cell Viability. Dimerization of ephrinB2-Fc ligand is necessary in order to fully activate EphB4 receptor; hence ephrinB2-Fc and Fc were preclustered with goat antihuman IgG at 1:3 concentration for 1 hour at room temperature prior to experiments.

Cells at $2.5 \times 10^{3}$ concentration were seeded into each well of a 24-well plate. Twenty four hours later, cells were incubated with medium containing 1,3 or $10 \mu \mathrm{g} / \mathrm{mL}$ preclustered ephrinB2-Fc or control Fc.

Cell viability was measured on days 3 and 6 , when cells were in sub- and postconfluent state, respectively. Fresh medium containing ephrinB2-Fc was replaced on day 3 for the 6-day assay.

Cells were rinsed with PBS at the indicated times and fresh medium was replaced. Alamar-blue was added to each 
well containing fresh medium with $10 \%$ FBS in an amount equal to $10 \%$ of volume in each well and was incubated for 3 hours. Aliquots of $200 \mu \mathrm{L}$ were dispensed in 96-well plates and absorbance was measured at 570 and $600 \mathrm{~nm}$ using a plate reader. Cells plus alamar-blue containing no test agent were used as positive growth control. Difference between percentage reduction of ephrinB2-Fc and Fc treated groups were then calculated based on equation (2) or online calculator.

Percent difference of dye reduction

$$
=\frac{(117216 \times A 1)-(80586 \times A 2) \times 100}{(117216 \times P 1)-(117216 \times P 2)}
$$

$A 1$ and $A 2$ are observed absorbance of test wells at 570 and $600 \mathrm{~nm}$, while $P 1$ and $P 2$ are observed absorbance of positive growth control, which consisted of cells plus alamar-blue but no test agent.

2.5. Cell Behavior on Matrigel. Matrigel was thawed on ice and $100 \mu \mathrm{L}$ solution was evenly added to each well of ice cold 24-well plate and was kept in incubator for 30 minutes. A suspension of $10^{5}$ cells plus solutions of 3 and $10 \mu \mathrm{g} / \mathrm{mL}$ preclustered ephrinB2-Fc or PBS as negative control were added to each well in a total volume of $400 \mu \mathrm{L}$, and plate was incubated for 24 hours. Cell behavior for tubular formation was observed and photographed through inverted light microscope.

2.6. Statistical Analysis. Difference between ephrinB2-Fc treated and non-treated groups was analyzed by student$t$-test. Analysis of variance followed by Post-Hoc test was performed to compare survival between various doses. Data are represented as average \pm S.E.M and $P<0.05$ was considered as significant.

\section{Results}

3.1. Optimal Conditions for Alarmar-Blue Viability Assay. Percentage reduction of alamar-blue after incubation with MDA-MB-231 cell line was measured at different cell densities and incubation periods, and data was plotted against various cell concentrations for each time point. The curve was linear between $5 \times 10^{3}$ and $2 \times 10^{4} \mathrm{cell} / \mathrm{mL}$ (Figure 1). Based on these results and a pilot test to determine optimal cell density for seeding cells in 24 -well plates, $2.5 \times 10^{3}$ cell $/ \mathrm{mL}$ concentration was chosen for cells to reach confluence not sooner than 4 days. Incubation time of 3 hours with alamar-blue was found to be optimal for calculation of percentage reduction. These values were chosen for future experiments with alamar-blue.

3.2. EphB4 Stimulation Decreases Cancer Cell Viability in a Dose and Time Dependent Manner. Analysis of receptor phosphorylation following stimulation via clustered ephrinB2 showed that EphB4 receptor was active in MDAMB-231 breast cancer cell line (data not shown). To determine the effect of receptor activation on cell viability and growth,

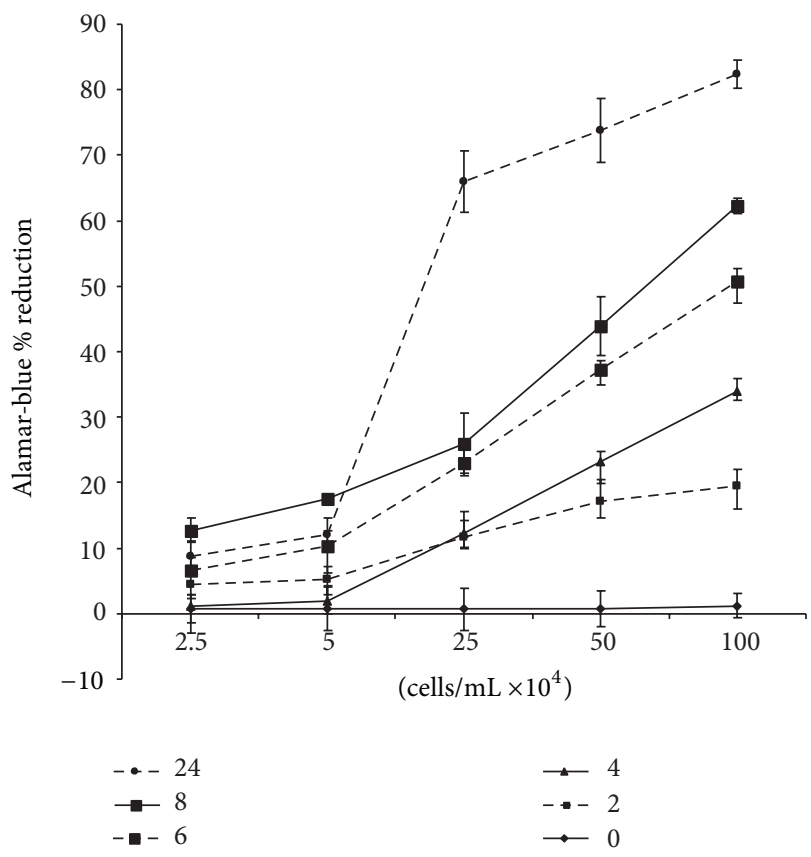

FIGURE 1: Alamar-blue percent reduction was measured at different time points and various cell dendities. Each line indicates duration of time for alamar-blue incubation with cells ( $0-24$ hours). As shown, plot is linear between $5 \times 10^{4}$ and $2 \times 10^{4} \mathrm{cell} / \mathrm{mL}$. Error bars show standard deviation of absorbance of 6 wells for three repeats.

cells were exposed to various doses of clustered ephrinB2Fc at different time points as discussed earlier. No significant reduction in cell viability was observed when cells were incubated with ephrinB2-Fc or Fc control for 3 days, neither when cells were about $60 \%$ confluent and were growing in monolayer state (Figure 2(a)). A significant decrease was detected after 6 days of incubation when cells had reached confluence and were continued to grow as a second layer; reduction in cell viability was more prominent in higher doses (Figure 2(b)). These results indicate that inhibition of cell growth mediated by EphB4 stimulation via ephrinB2 occurs only after longer incubation times.

3.3. EphrinB2-Fc Treatment Does Not Affect Cell Behavior on Matrigel. Matrigel is a commercial mixture of collagen and laminin which are the main constituents of extracellular matrix. Transformed and nontransformed cells display different morphologies on matrigel and this property that can be exploited to study the effect of antitumor agents on reversing the transform cell behavior. Normal cells such as MCF-10A form spherical bodies on matrigel, while transformed and invasive cells such as MDA-MD-231 breast cancer cell line invade matrigel and form tubular networks.

We tested if ephrinB2-Fc treatment can inhibit tubular network formation of MDA-MB-231 on matrigel. Cells were incubated on matrigel for 24 hours in the presence of preclustered ephrinB2-Fc (examining $3 \mu \mathrm{g} / \mathrm{mL}$ and $10 \mu \mathrm{g} / \mathrm{mL}$ concentrations). MDA-MB-231 cells invaded into matrigel and formed tubular networks. EphrinB2, at any of tested 


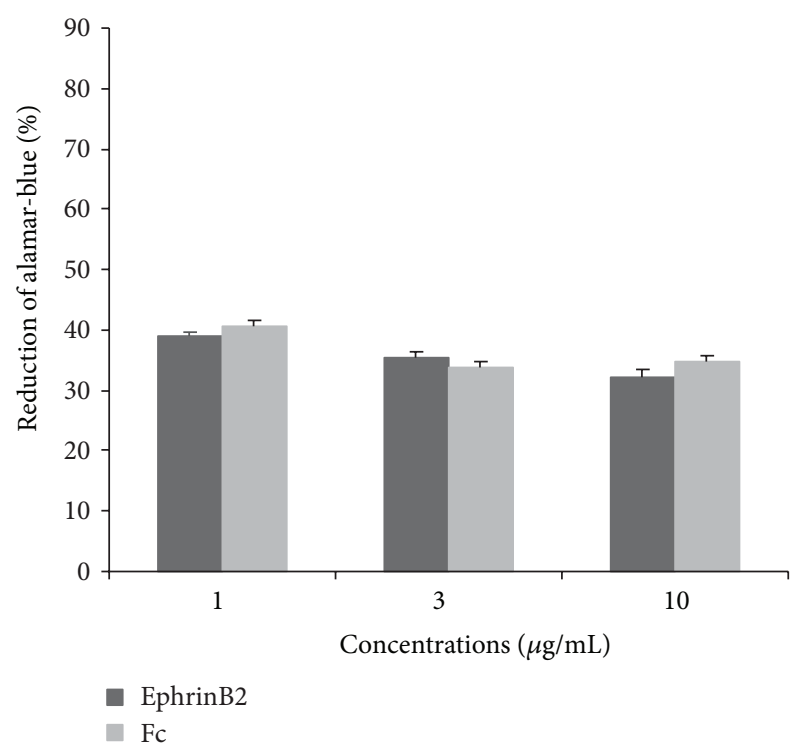

(a)

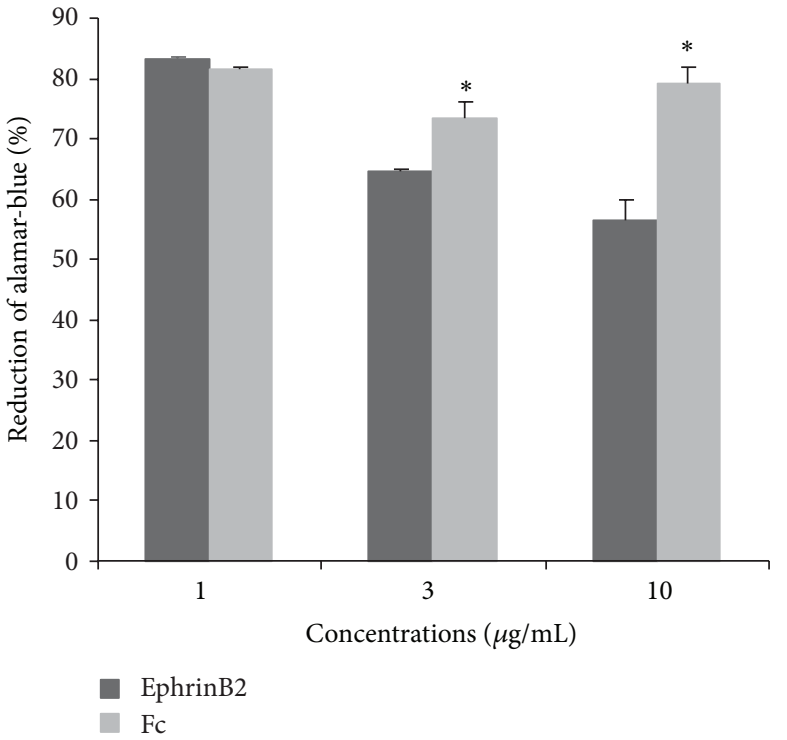

(b)

FIGURE 2: Cell viability measured after 3 and 6 days of treatment with ephrinB2-Fc or Fc. (a) Cell viability did not change following 3 days of incubation compared to control (ANOVA; $P>0.05$ ). (b) Six days of treatment with ephrinB2-Fc, however, significantly decreased cell viability at concentrations of $3 \mu \mathrm{g} / \mathrm{mL}$ and $10 \mu \mathrm{g} / \mathrm{mL}$ but not $1 \mu \mathrm{g} / \mathrm{mL}$. (ANOVA followed by Post-Hoc; $P<0.05$, marked with asterisk). Error bars represent mean \pm S.E.M of cell viability for three separate repeats.

doses; did not alter invasive behavior of cells as shown in Figure 3. If ephrinB2 treatment was to affect invasive behavior on matrigel, the tube formation was expected to be abrogated totally, and aggregated colonies were to be seen instead of tubules, as shown by Carles-Kinch et al. [19]. These results indicate that the observed decrease in cell viability mediated via EphB4 stimulation after 6 days is not likely due to specific inhibition of multi layer growth stage named anchorage independent growth.

\section{Discussion}

Eph family of receptors are interesting targets for molecular cancer therapy since they are mostly overexpressed in tumors and seem to be involved in tumor cell survival and proliferation, invasion, metastasis, and angiogenesis. Thus, designing agents to properly target them can induce tumor growth arrest by multiple mechanisms. However, rational drug design to thoroughly exploit their ability to inhibit tumor growth requires more accurate understanding of mechanisms underlying Eph/ephrin system signaling in cancer cells and their microenvironment.

In this study, we showed that long term incubation of MDA-MB-231 breast cancer cells with ephrinB2-Fc inhibited their growth when cells were growing in postconfluent state, and growth inhibition was more prominent in higher doses. A similar study by Carles-Kinch et al. revealed that use of activating antibodies against EphA2, another member of Eph family overexpressed in MDA-MB-231 cell line, led to inhibition of cell growth after reaching confluence as well as inhibiting tubular formation on matrigel [19].
Inhibition of cell growth after 6 days might be due to receptor downregulation and deletion from cell surface rather than inhibition of anchorage independent growth. This mechanism was seen in a similar study by Kumar et al. in which they showed that long term treatment of MCF-7 cells with ephrinB2 led to receptor downregulation and growth inhibition, similar to results from knock down of EphB4 [14]. On the other hand, with the conditions used in this study, ephrinB2-Fc treatment did not reduce formation of tubules and invasion. It is thus speculated that ephrinB2Fc is more likely an inhibitor of tumor cell viability and reduction of tumor invasion may require higher doses or in vivo conditions where inhibition of angiogenesis leads to suppression of invasion and metastasis.

Recent studies have uncovered interesting aspects of Eph receptors in breast cancer yet exact roles of Eph/ephrin in promotion or suppression of tumors remains unclear. Dual roles of tumor promotion and suppression (Figure 4) have been assigned to this family, possibly based on presence or absence of their cognate ligand [13]. Accordingly, EphB4 overexpression has been correlated with increased tumor stage and poor survival [20]. Therefore, EphB4 may serve as a survival factor for breast cancer cells possibly due to constitutive activation or crosstalk with other oncogenic growth factor receptors such as EGFR family [14]. In contrast, EphB4 activation by its ligand, ephrinB2, in breast cancer cells has been shown to inhibit tumor promotion and invasion through activation of Abl-Crk pathway that was consistant with our results [17].

Yet the scenario seems to be much more complicated to describe the opposing effects of EphB4 stimulation solely by presence or absence of ephrinB2. In this regard, cell context 


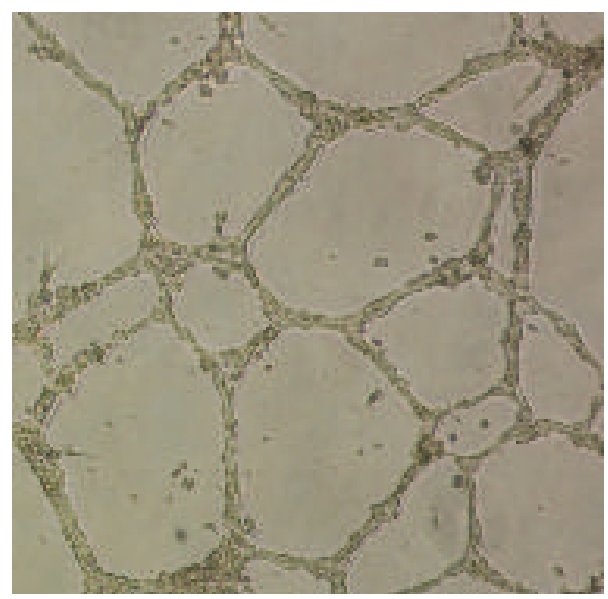

(a)

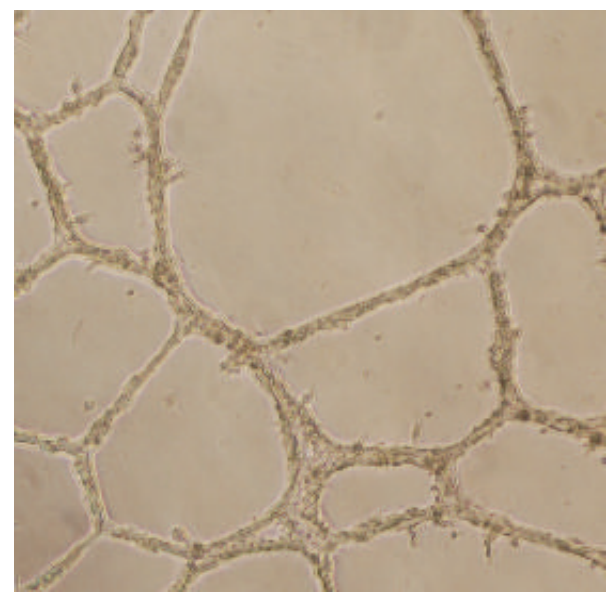

(b)

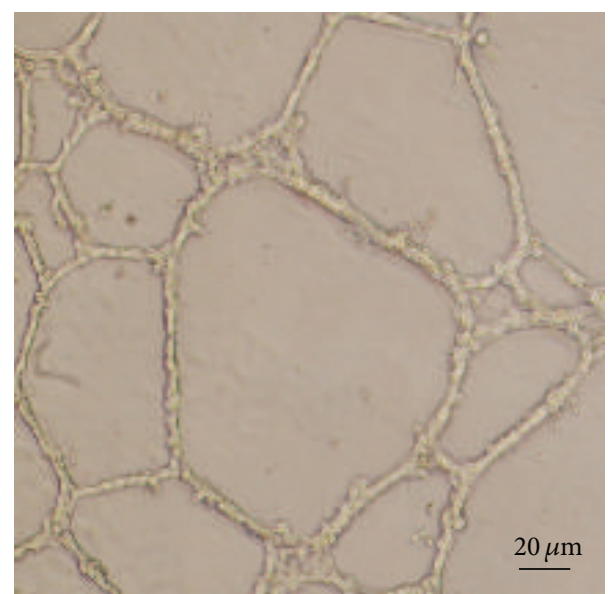

(c)

FIGURE 3: Formation of tubular network by MDA-MB-231 cells was tested on matrigel in presence and absence of ephrinB2-Fc. Cells were treated with (a) PBS, (b) $3 \mu \mathrm{g} / \mathrm{mL}$ ephrinB2-Fc and (c) $10 \mu \mathrm{g} / \mathrm{mL}$ ephrinB2-Fc. Treatment with ephrinB2-Fc was expected to totally abrogate tubular formation and form aggregated colonies, yet it was surprisingly observed that tubular formation was not inhibited by ephrinB2-Fc treatment compared to PBS. Figures were inspected at 100x magnification and scale bars represent $20 \mu \mathrm{m}$.

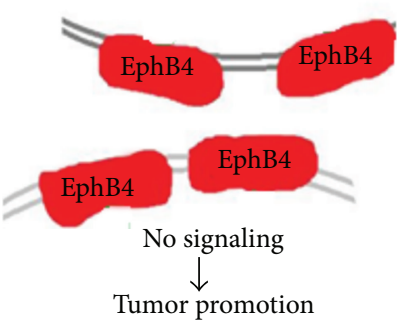

(a)

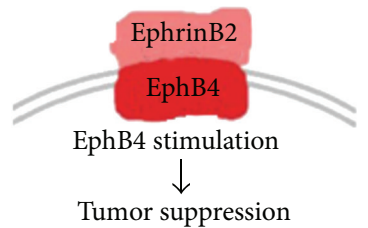

(b)
FIGURE 4: (a) Eph receptor stimulation is lost during carcinogenesis due to down-regulation of ephrin ligands. (b) It was shown in our study that representation of exogenous ephrinB2 to re-establish balance between receptor and ligand signaling is able to exhibits anti-tumor effects.

also seems to play a pivotal role in determining effects on tumor promotion or suppression as well. This assumption has been supported recently in a study by Xiao et al. in which they demonstrated that ephrinB2-Fc treatment leads to growth inhibition in HUVEC cell, while the same stimulation leads to growth promotion in MCF-7 cells [21]. Paradoxes about the role of EphB4 receptor in breast cancer go even further and are evident in signaling mechanisms assigned for them as well. Kumar et al. have previously shown that EphB4 stimulation via ephrinB2 leads to activation of PI3 K/Akt pathway- and a 4-day treatment of MCF-7 cells with the ligand led to cell death due to receptor down-regulation [14]. On the other hand, Xiao et al. reported an increase in growth of MCF-7 cells following ephrinB2 treatment due to activation of Ras/Erk pathway after 6 days [21]. Such discrepancies in the same context may be due to variations in experimental conditions such as ligand dose and timing of receptor stimulation which was shown to be effective in our study as well and the extent of ligand clustering with anti-Fc antibody or sensitivity of viability assays used. Dependence of response from growth inhibition to promotion upon different stimulation conditions further sophisticates smart drug design for targeting EphB4 in breast cancer and therefore necessitates further studies to more accurately address the advantage of EphB4 activation over EphB4 inhibition in various cancers.

\section{Conflict of Interests}

The authors have no conflict of interests.

\section{Authors' Contribution}

Mona Moshayedi and Farnaz Barneh contributed equally to this study.

\section{Financial Support}

This study was supported by grants from Research Council, Isfahan University of Medical Sciences. 


\section{Acknowledgment}

The authors thank Nasim Dana (Applied Physiology Research Center) for kindly providing matrigel.

\section{References}

[1] D. Hanahan and R. A. Weinberg, "The hallmarks of cancer," Cell, vol. 100, no. 1, pp. 57-70, 2000.

[2] J.-P. Himanen, N. Saha, and D. B. Nikolov, "Cell-cell signaling via Eph receptors and ephrins," Current Opinion in Cell Biology, vol. 19, no. 5, pp. 534-542, 2007.

[3] E. B. Pasquale, "Eph receptors and ephrins in cancer: bidirectional signalling and beyond," Nature Reviews Cancer, vol. 10, no. 3, pp. 165-180, 2010.

[4] V. C. Dodelet and E. B. Pasquale, "Eph receptors and ephrin ligands: embryogenesis to tumorigenesis," Oncogene, vol. 19, no. 49, pp. 5614-5619, 2000.

[5] E. B. Pasquale, "Eph receptor signalling casts a wide net on cell behaviour," Nature Reviews Molecular Cell Biology, vol. 6, no. 6, pp. 462-475, 2005.

[6] E. B. Pasquale, "Eph-ephrin bidirectional signaling in physiology and disease," Cell, vol. 133, no. 1, pp. 38-52, 2008.

[7] N. Cheng, D. M. Brantley, and J. Chen, "The ephrins and Eph receptors in angiogenesis," Cytokine and Growth Factor Reviews, vol. 13, no. 1, pp. 75-85, 2002.

[8] D. Vaught, D. M. Brantley-Sieders, and J. Chen, "Eph receptors in breast cancer: oles in tumor promotion and tumor suppression," Breast Cancer Research, vol. 10, no. 6, article 217, 2008.

[9] N. K. Noren, M. Lu, A. L. Freeman, M. Koolpe, and E. B. Pasquale, "Interplay between EphB4 on tumor cells and vascular ephrin-B2 regulates tumor growth," Proceedings of the National Academy of Sciences of the United States of America, vol. 101, no. 15, pp. 5583-5588, 2004.

[10] G. Martiny-Baron, T. Korff, F. Schaffner et al., "Inhibition of tumor growth and angiogenesis by soluble EphB4," Neoplasia, vol. 6, no. 3, pp. 248-257, 2004.

[11] N. Kertesz, V. Krasnoperov, R. Reddy et al., "The soluble extracellular domain of EphB4 (sEphB4) antagonizes EphB4EphrinB2 interaction, modulates angiogenesis, and inhibits tumor growth," Blood, vol. 107, no. 6, pp. 2330-2338, 2006.

[12] D. M. Brantley-Sieders, A. Jiang, K. Sarma et al., "Eph/ephrin profiling in human breast cancer reveals significant associations between expression level and clinical outcome," PLoS One, vol. 6, no. 9, Article ID e24426, 2011.

[13] R. Rutkowski, I. Mertens-Walker, J. E. Lisle, A. C. Herington, and S.-A. Stephenson, "Evidence for a dual function of EphB4 as tumor promoter and suppressor regulated by the absence or presence of the ephrin-B2 ligand," International Journal of Cancer, vol. 131, no. 5, pp. E614-E624, 2012.

[14] S. R. Kumar, J. Singh, G. Xia et al., "Receptor tyrosine kinase EphB4 is a survival factor in breast cancer," American Journal of Pathology, vol. 169, no. 1, pp. 279-293, 2006.

[15] N. K. Noren and E. B. Pasquale, "Paradoxes of the EphB4 receptor in cancer," Cancer Research, vol. 67, no. 9, pp. 39943997, 2007.

[16] P. Kaenel, M. Mosimann, and A. C. Andres, "The multifaceted roles of Eph-ephrin signaling in breast cancer," Cell Adhesion \& Migration, vol. 6, no. 2, pp. 138-147, 2012.

[17] N. K. Noren, G. Foos, C. A. Hauser, and E. B. Pasquale, "The EphB4 receptor suppresses breast cancer cell tumorigenicity through an Abl-Crk pathway," Nature Cell Biology, vol. 8, no. 8, pp. 815-825, 2006.

[18] S. Al-Nasiry, N. Geusens, M. Hanssens, C. Luyten, and R. Pijnenborg, "The use of Alamar Blue assay for quantitative analysis of viability, migration and invasion of choriocarcinoma cells," Human Reproduction, vol. 22, no. 5, pp. 1304-1309, 2007.

[19] K. Carles-Kinch, K. E. Kilpatrick, J. C. Stewart, and M. S. Kinch, "Antibody targeting of the EphA2 tyrosine kinase inhibits malignant cell behavior," Cancer Research, vol. 62, no. 10, pp. 2840-2847, 2002.

[20] B. P. Fox and R. P. Kandpal, "Invasiveness of breast carcinoma cells and transcript profile: eph receptors and ephrin ligands as molecular markers of potential diagnostic and prognostic application," Biochemical and Biophysical Research Communications, vol. 318, no. 4, pp. 882-892, 2004.

[21] Z. Xiao, R. Carrasco, K. Kinneer et al., "EphB4 promotes or suppresses Ras/MEK/ERK pathway in a context-dependent manner: implications for EphB4 as a cancer target," Cancer Biology \& Therapy, vol. 13, no. 8, 2012. 


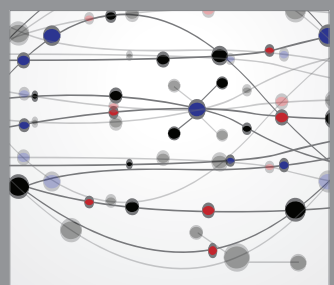

The Scientific World Journal
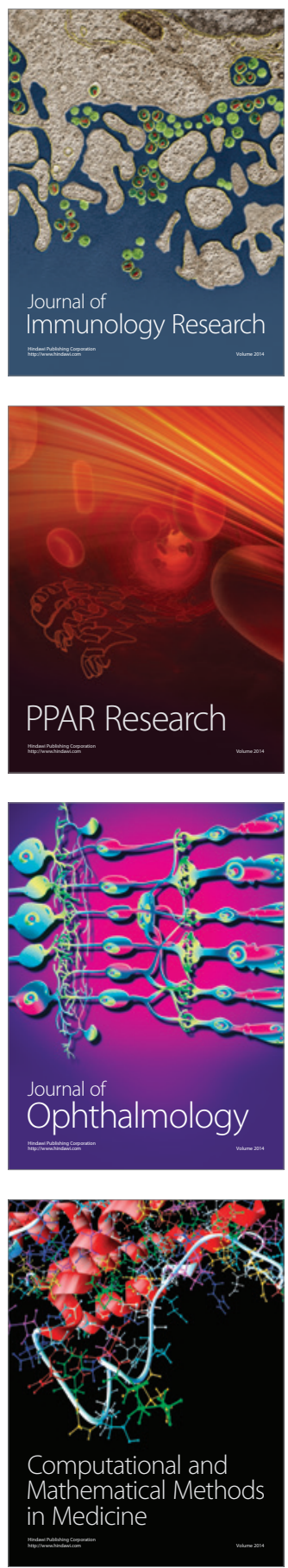

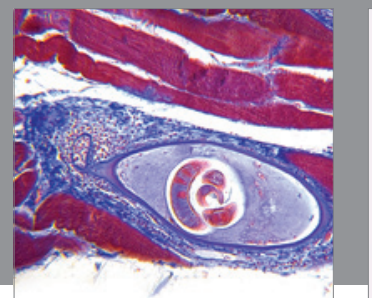

Gastroenterology

Research and Practice
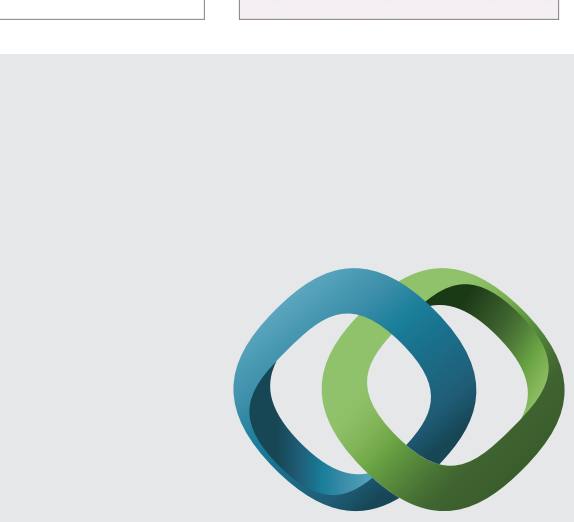

\section{Hindawi}

Submit your manuscripts at

http://www.hindawi.com
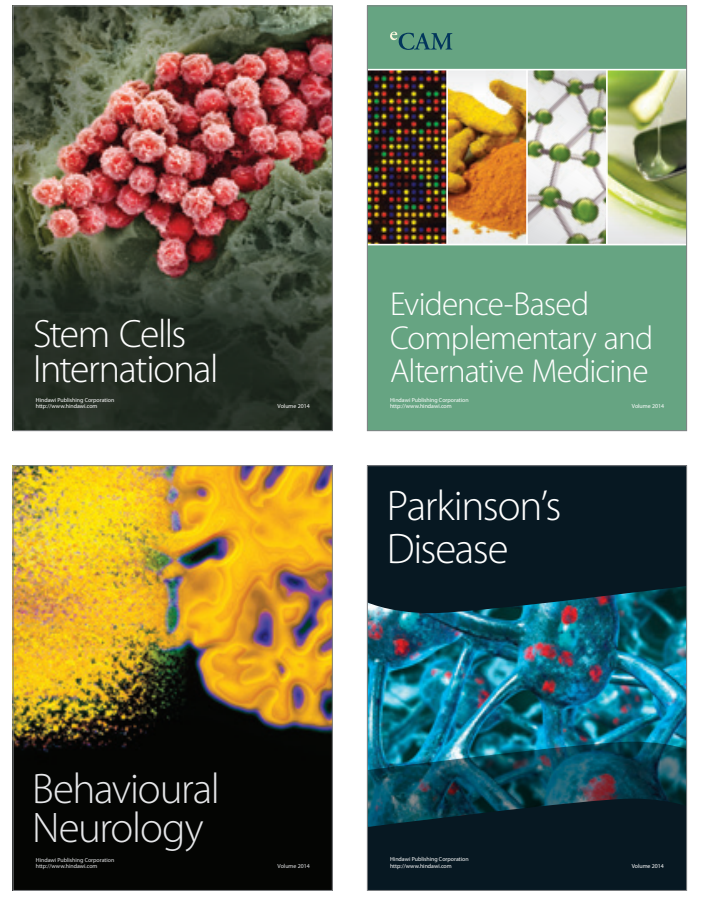
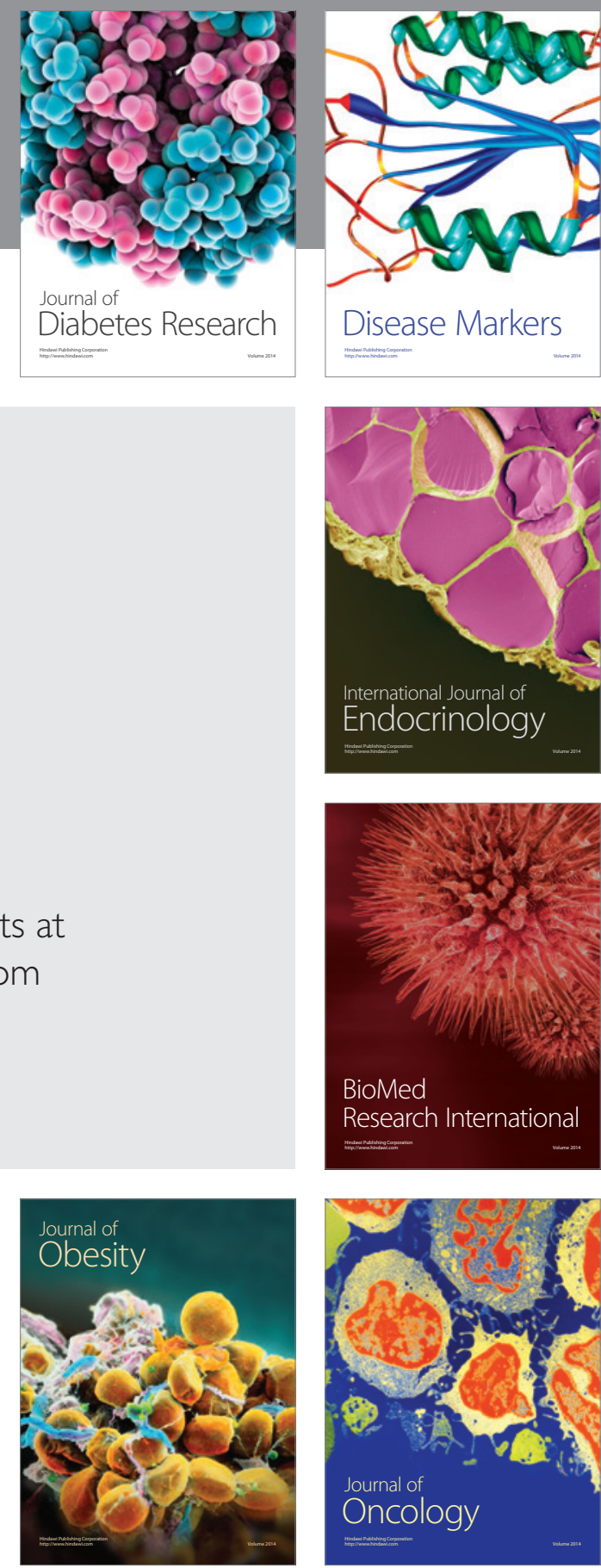

Disease Markers
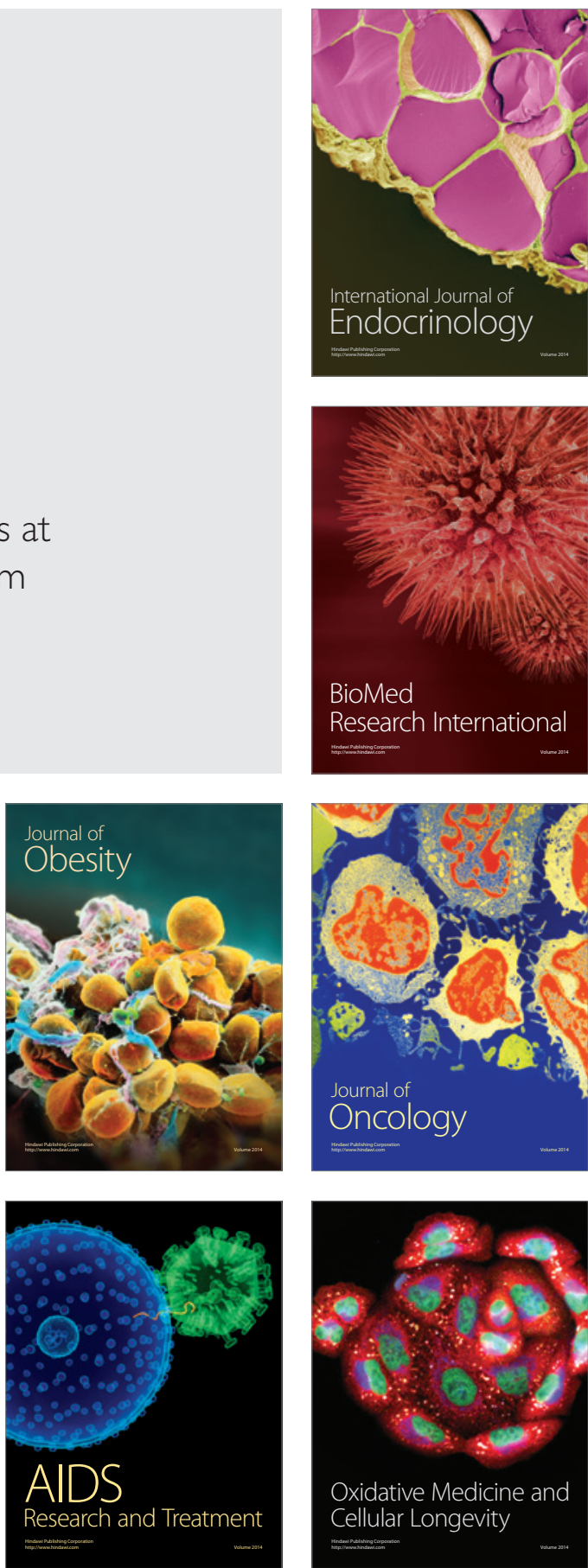